\title{
Metallicity gradients in the Milky Way
}

\author{
Walter J. Maciel and Roberto D. D. Costa \\ University of São Paulo, Astronomy Department, \\ Rua do Matão 1226, Cidade Universitária, São Paulo SP, CEP 05509-0900, Brazil \\ email: maciel@astro.iag.usp.br, roberto@astro.iag.usp.br
}

\begin{abstract}
Radial metallicity gradients are observed in the disks of the Milky Way and in several other spiral galaxies. In the case of the Milky Way, many objects can be used to determine the gradients, such as HII regions, B stars, Cepheids, open clusters and planetary nebulae. Several elements can be studied, such as oxygen, sulphur, neon, and argon in photoionized nebulae, and iron and other elements in cepheids, open clusters and stars. As a consequence, the number of observational characteristics inferred from the study of abundance gradients is very large, so that in the past few years they have become one of the main observational constraints of chemical evolution models. In this paper, we present some recent observational evidences of abundance gradients based on several classes of objects. We will focus on (i) the magnitude of the gradients, (ii) the space variations, and (iii) the evidences of a time variation of the abundance gradients. Some comments on recent theoretical models are also given, in an effort to highlight their predictions concerning abundance gradients and their variations.
\end{abstract}

Keywords. stars: abundances, ISM: abundances, photoionized nebulae, Galaxy: abundances

\section{Introduction}

Radial metallicity gradients are observed in the disks of many galaxies, including the Milky Way, galaxies of the Local Group and other objects. Current research topics include the determination of (i) the magnitude of the gradients, (ii) any space variations along the disk and (iii) possible time variations during the evolution of the host galaxy. In this paper, we present some recent observational evidences of radial abundance gradients. Our main focus is the Milky Way, but it will be shown that the analysis of some objects in the Local Group, particularly M33, is useful in order to study the main properties of the gradients in our own Galaxy. A brief discussion of some recent theoretical models is also given, in an effort to highlight their predictions concerning the radial abundance gradients and their variations. Some recent reviews and general papers on abundance gradients include: Freeman (2009), Maciel \& Costa (2009), Rudolph et al. (2006), and Stasińska (2004). Theoretical models are discussed by a number of people, including $\mathrm{Fu}$ et al. (2009), Magrini et al. (2009a), Cescutti et al. (2007), Mollá and Díaz (2005), Chiappini et al. (2003, 2001, 1997), Hou et al. (2000), and Gensler (these proceedings). Recent discussions on azimuthal and vertical gradients, not treated here, can be found in Davies et al. (2009) and Ivezić et al. (2008).

\section{Abundance gradients in the Milky Way}

\subsection{Cepheids}

Cepheid variables are possibly the most accurate indicators of abundance gradients in the Milky Way. Since the work of Andrievsky and collaborators (cf. Andrievsky et al. 2002a, 2002b, 2002c, 2004, Luck et al. 2003), several papers have dealt with these objects in order to study not only the magnitudes of the present-day gradients, but also the detailed 
behaviour of the abundances along the galactic disk. The main reason is that cepheids are bright enough to be observed at large distances, so that accurate distances and spectroscopic abundances of several elements can be obtained, which in principle allow the determination of radial variations of the gradients better than any other indicator. The ages of these objects are generally under $200 \mathrm{Myr}$ (cf. Maciel et al. 2005), so that the measured gradients can be safely considered as present-day gradients. Recent work on Cepheids include Lemasle et al. (2008), Pedicelli et al. (2009), and Romaniello et al. (2008). Lemasle et al. (2008) obtained high-resolution spectroscopic iron abundances for galactic cepheids for which accurate distances were determined based on near-infrared photometry. The abundances were determined within 0.12 dex, while the distances, based on a near-infrared period-luminosity relation, are expected to be accurate within $0.5 \mathrm{kpc}$ in average. In order to improve the sampling process, additional objects were included. The average gradient obtained in the range $5-17 \mathrm{kpc}$ is $d[\mathrm{Fe} / \mathrm{H}] / d R=-0.052 \pm 0.003 \mathrm{dex} /$ kpc. A better solution proposed by the authors includes a change of slope, in the sense that the gradient is steeper in the inner galaxy, with a flattened gradient of $-0.012 \pm$ $0.014 \mathrm{dex} / \mathrm{kpc}$ in the outer galaxy. In this region the abundances show an increased spread, as compared with the inner galaxy. The change of slope occurs at about $R \simeq 10$ $\mathrm{kpc}$, farther away than the solar radius, located at $R=8.5 \mathrm{kpc}$. These results have been largely confirmed by the recent work of Pedicelli et al. (2009), in which again a large sample from different sources was considered with a new photometric metallicity calibration. These results suggest a gradient of $-0.051 \pm 0.004 \mathrm{dex} / \mathrm{kpc}$ for the whole sample, or $-0.130 \pm 0.015 \mathrm{dex} / \mathrm{kpc}$ for the inner sample $(R<8 \mathrm{kpc})$ and $-0.042 \pm 0.004 \mathrm{dex} /$ kpc for the outer Galaxy.

\subsection{HII regions}

Concerning HII regions, several determinations have been presented in the last few years, which include Deharveng et al. (2000), Esteban et al. (2005), Rudolph et al. (2006) and Quireza et al. (2006). Deharveng et al. (2000) obtained an oxygen gradient of -0.04 $\mathrm{dex} / \mathrm{kpc}$ in the range $5-15 \mathrm{kpc}$, with no indication of flattening further out in the disk. Pilyugin et al. (2003) compiled spectra of $13 \mathrm{HII}$ regions in the range $7-14 \mathrm{kpc}$ with available [OIII] $\lambda 4363$ measurements, and recomputed the oxygen abundances from the data by Shaver et al. (1983), obtaining a gradient of -0.051 dex/kpc. Esteban et al. (2005) obtained echelle spectrophotometry of $8 \mathrm{HII}$ regions in the range $6-10 \mathrm{kpc}$, and derived carbon and oxygen abundances from recombination lines. The oxygen gradient obtained is $-0.044 \pm 0.010 \mathrm{dex} / \mathrm{kpc}$. More recently, Rudolph et al. (2006) used both infrared and optical data to study abundance gradients in a sample of 117 HII regions. The data include both new results and a reanalysis of previous material. The best fit to optical data corresponds to a gradient of $-0.060 \mathrm{dex} / \mathrm{kpc}$. For the infrared data a gradient of $-0.041 \mathrm{dex} / \mathrm{kpc}$ was obtained. Quireza et al. (2006), determined the electron temperature gradient from high-precision radio recombination line and continuum measurements for over a hundred HII regions, calibrated in terms of the oxygen abundance gradient. The derived slope obtained using a relation between the electron temperature and the oxygen abundances by Shaver et al. (1983) is $-0.043 \pm 0.007 \mathrm{dex} / \mathrm{kpc}$, in good agreement with the previous work by Deharveng et al. (2000) and Esteban et al. (2005).

From these results it is difficult to establish whether or not the gradients flatten out at large galactocentric distances. However, studies of HII regions in spiral galaxies are consistent with essentially constant abundances in the outer parts of these objects, as for example in the recent work of Bresolin et al. (2009a) on the extended disk of M83, in which a flat oxygen gradient was obtained beyond the R25 isophotal radius, regardless of the abundance indicator used. 


\subsection{Stars and Open Clusters}

Apart from Cepheids, other field stars can be used in order to analyze the abundance gradients in the Milky Way. For open cluster stars, both the metallicity and the distances are well determined, and they have a reasonably large age span, so that they can be used to investigate both the spatial and temporal changes in the gradients. However, the age determinations may depend on the calibration used. Work up to 2007 is summarized by Cescutti et al. (2007), where gradients of O, Mg, Si, S, and Ca are discussed. Several objects have been considered, comprising Cepheids, O, B stars, red giants, and two samples of open clusters. The main feature which is common to all data is a change of slope at large galactocentric radii, roughly $R>10 \mathrm{kpc}$, which characterizes the flattening of the gradients in the outer Galaxy. Theoretical models are also discussed, which assume an inside-out formation for the galactic disk with a time scale of 7 Gyr for the thin disk in the solar vicinity and a shorter timescale of $0.8 \mathrm{Gyr}$ for the galactic halo. The inside-out scenario is apparently a necessary condition to explain the present-day gradients and the variation of the star formation rate with the galactocentric radius. In fact, recent models by Colavitti et al. (2009) conclude that all disk constraints cannot be simultaneously satisfied unless an inside-out formation of the galactic disk is assumed.

More recent work on open clusters (Sestito et al. 2008, Magrini et al. 2009a) generally confirm these findings, using larger samples. A steep negative gradient is found for the inner Galaxy up to about 10-11 kpc, with a flat distribution in the outer Galaxy. A comparison is made of these results with the earlier work by Friel et al. (2002) based on low-resolution spectroscopy. Although the error bars are larger, there is not much difference relative to the high-resolution data for the inner Galaxy. The slopes are -0.17 dex/ $\mathrm{kpc}$ and $-0.09 \mathrm{dex} / \mathrm{kpc}$ for these samples, considering only the inner region. It is doubtful whether the difference is meaningful at this stage. In the outer Galaxy, an essentially flat gradient is observed for the new sample.

In view of the age span of the open clusters, they are ideally suited to study any time variations of the gradients. Magrini et al. (2009a) considered a sample of open clusters with high-resolution data, in the range $7-22 \mathrm{kpc}$, with estimated ages in the range $30 \mathrm{Myr}$ to 11 Gyr. The authors present plots of the gradients according to the age interval of the clusters for $\mathrm{Fe}, \mathrm{Cr}, \mathrm{Ni}, \mathrm{Si}, \mathrm{Ca}$, and $\mathrm{Ti}$, with a generally similar behaviour. Also, the ratios of these elements to Fe are essentially constant, suggesting that the derived gradients are similar within the uncertainties. Again, a steep gradient was found for the inner Galaxy $(R<12 \mathrm{kpc})$, with a flattening outwards. The flattening can be observed in all age brackets, but is especially clear in the sample with 4-11 Gyr. Considering the gradients obtained by adopting three different galactocenctric ranges leads to the conclusion that the gradients are approximately constant or slightly flattening with time. However, from the slopes of the inner Galaxy, this conclusion is probably a conservative one, as the indication of a flattening of the gradients seems clear.

Recent large surveys of galactic stars are also being used to derive abundance gradients, mainly based on the $[\mathrm{Fe} / \mathrm{H}]$ ratio. Some examples can be seen in the presentations by B. Nordström on the Geneva-Copenhagen survey and by C. Boesche on the RAVE project (cf. the conference site of The Milky Way and the Local Group: Now and in the GAIA Era, Heidelberg, 2009).

\subsection{Planetary Nebulae}

The analysis of abundance gradients from planetary nebulae (PN) is hampered by some aspects related to these objects: first, the distances to the galactic nebulae are often uncertain, and statistical scales have to be used in order to have a sizable sample; second, the progenitors of the planetary nebulae have a wide age span, as in the case of the open 

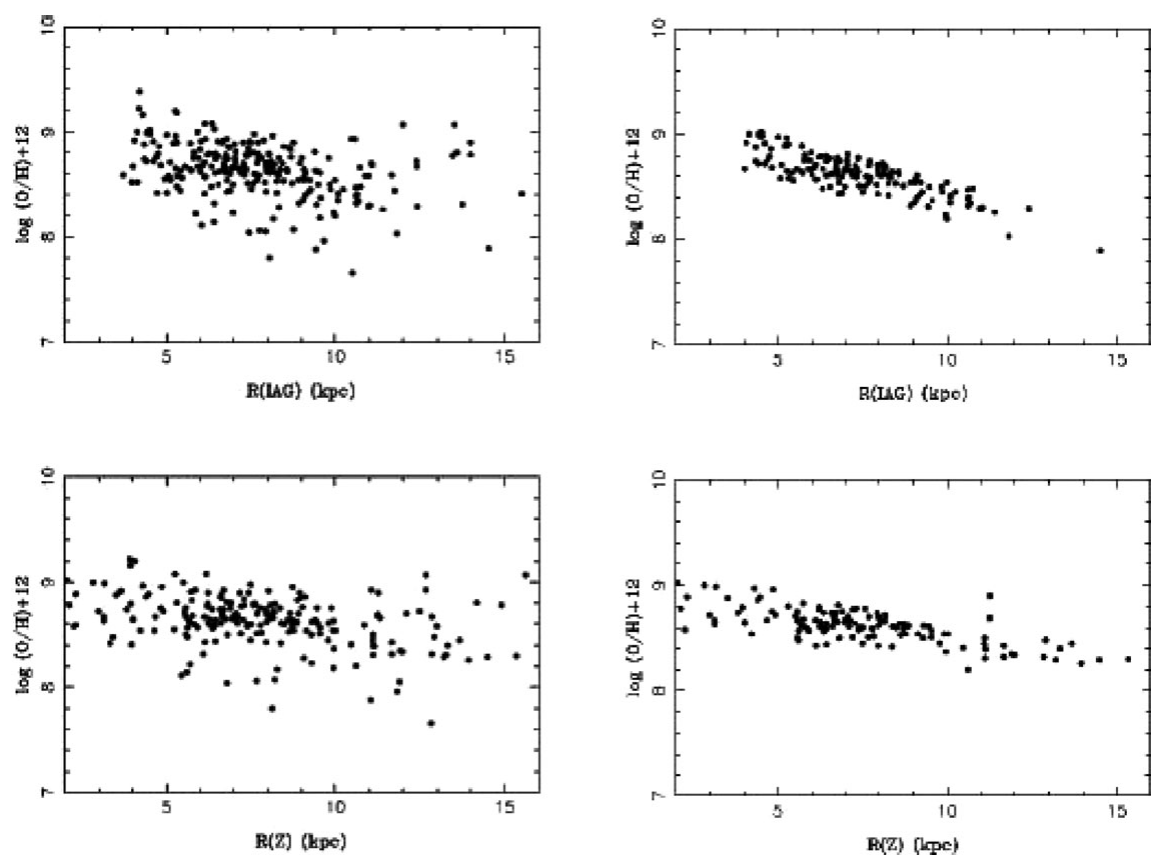

Figure 1. The O/H radial gradient from PN. Top: Distances from the IAG/USP group, Bottom: Distances from Stanghellini et al. (2008). Left: CSPN with ages in the range 2-10 Gyr; Right: CSPN with ages in the range 4-6 Gyr.

clusters, ranging from about 1 Gyr to about 8 Gyr (cf. Maciel et al. 2009), so that any time variation of the gradients would have to be taken into account. On the other hand, abundances of elements such as $\mathrm{O}, \mathrm{Ne}, \mathrm{S}$, and Ar can be obtained within about 0.2 dex in average, which is probably lower than the abundance spread at a given galactocentric radius. The results presented in the last couple of years have been rather contradictory. Pottasch and collaborators (cf. Pottasch and Bernard-Salas 2006) have presented accurate abundance data based both on optical and infrared measurements from ISO, which do not need the consideration of the usually uncertain ionization correction factors (ICF), since more ionized species can be observed. As a result, the derived abundances are expected to be more accurate than in the case of the traditional plasma diagnostic method. The results suggest the presence of a strong negative gradient similar to the ones observed in HII regions and early type stars. For the $\mathrm{O} / \mathrm{H}$ ratio a slope of -0.085 dex/ $\mathrm{kpc}$ was found. For most of the elements considered, which are $\mathrm{O}, \mathrm{Ne}, \mathrm{S}$, and Ar, the predicted abundances at the solar radius obtained by taking into account the observed gradients match exactly the solar abundances. In a more recent work (Gutenkunst et al. 2008), a larger sample was considered, including bulge nebulae. It can be concluded that the gradient flattens out near the galactic bulge, a result also obtained by Cavichia et al. (2009). In contrast with these results, Stanghellini et al. (2006) studied a sample of galactic PN with abundances derived from the traditional optical plasma diagnostic method, obtaining a flat gradient for oxygen and neon, based on a simple linear fit. However, from what we have seen in the previous sections, it seems clear that the gradients flatten out in the outer Galaxy, so that using a single fit for the whole disk may be misleading, especially if the inner Galaxy, where the gradient is steeper, is undersampled as is the case here. Moreover, the data in this sample located in the region around the solar circle clearly show some evidence of a steeper gradient, so that a flat gradient for the 


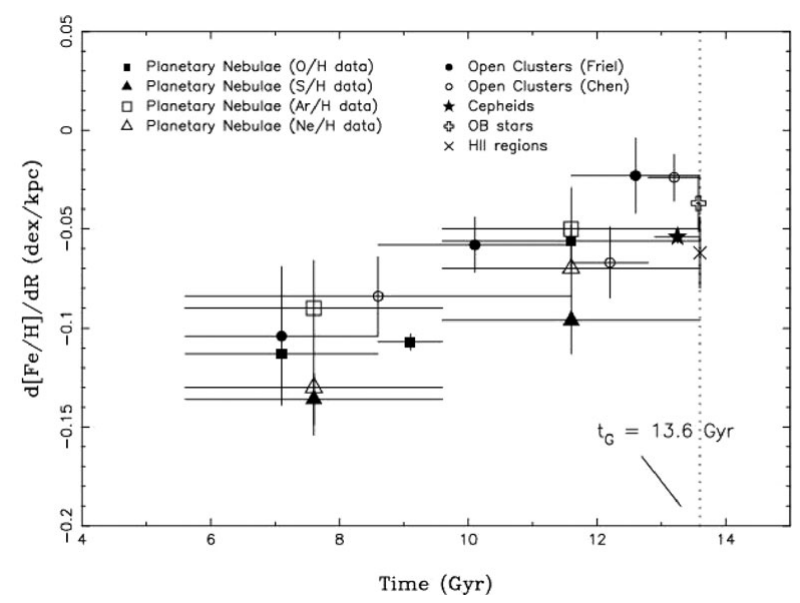

Figure 2. Time variation of the radial abundance gradient (Maciel \& Costa 2009).

whole disk is probably incorrect. A more detailed analysis was made by Perinotto and Morbidelli (2006), who considered a larger and more complete sample of PN for which the abundances were recalculated in a homogeneous way. The results also suggest relatively flat gradients $(<-0.04 \mathrm{dex} / \mathrm{kpc})$ for oxygen, but a careful analysis of their data shows that the uncertainties in the distances, coupled with a possible time variation of the gradients, may wash out the gradients, so that a careful selection of the objects must be made. This can be seen by considering the oxygen gradients for PN of sets $\mathrm{A}$ and $\mathrm{B}$, defined as follows: set A includes 131 objects whose abundances are considered by the authors as the most reliable, and set B is a control sample, containing all PN abundances published between 2000 and 2005, with about 200 objects. In order to avoid any bias due to the adopted distances, Perinotto and Morbidelli (2006) considered four different statistical scales. Considering for example the results corresponding to the distances by Cahn et al. (1992), a gradient is apparent from set A, while set B presents a flat distribution, suggesting that the uncertainties in the abundances contribute to erase any existing gradients. Some indication of the existence of a time variation of the gradients, in the sense that the present-day gradient is flatter than in the past can also be observed from the results by Perinotto and Morbidelli (2006). They have analyzed separately the PN according to the Peimbert types, in which Type I are expected to be younger objects, while Type III are older nebulae, generally located at higher distances from the galactic plane and with a larger peculiar velocity. Type II are intermediate age objects in this scheme. According to Perinotto and Morbidelli (2006), Type I objects do not show any gradients in both sets, while Type II and III show measurable, albeit low, gradients. Also, for the distance scales with a meaningful sample, the gradients of Type III objects are larger than for Type II PN.

A different approach has been taken by the IAG/USP group (Maciel et al. 2003, 2005, 2006, 2009, Maciel \& Costa 2009). Here an effort has been made to divide the PN sample into age groups, as was done for the open clusters by Magrini et al. (2009a), so that any time variation of the gradients could be appropriately taken into account. Several methods have been developed to obtain the age distribution of the PN central stars. Fig. 1 shows the $\mathrm{O} / \mathrm{H}$ gradient for disk objects with ages of 2-10 Gyr (left) and 4-6 Gyr (right), using the distance scale adopted by our IAG Basic Sample (top), or the distance scale by Stanghellini et al. (2008) (bottom). The effect of restricting the age interval is 

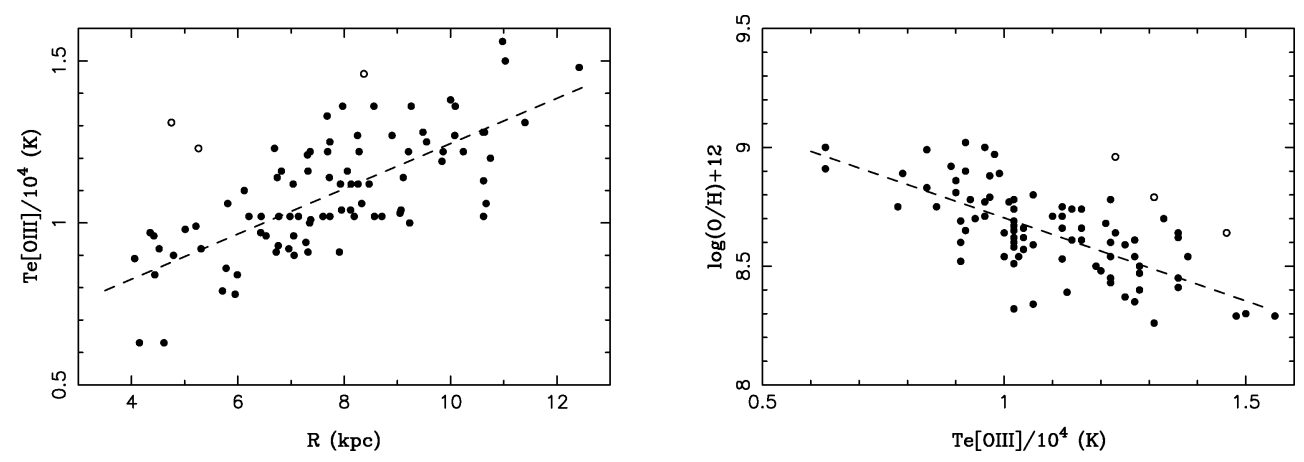

Figure 3. Left: The [OIII] electron temperature gradient from planetary nebulae. Right: The correlation between the electron temperatures and oxygen abundances (Maciel et al. 2007).

clear in both figures. Taking into account the age distribution of the PN progenitor stars (Maciel et al. 2009), it is possible to separate the PN sample according to their ages (young, intermediate, old), so that an estimate of the time variation of the gradients can be obtained. This is shown in Fig. 2 (Maciel \& Costa 2009), where other objects are also considered, namely open clusters, cepheids, OB stars and HII regions. As a conclusion, data from planetary nebulae support the flattening of the gradients near the bulge-disk interface and at large galactocentric distances. However, anticentre nebulae are difficult to observe, and the problem of the distances is still a complicating factor. A considerable improvement is expected with the advent of GAIA. As for the time variation of the gradients, a conservative conclusion at this stage is that either they have not changed very much in the last 6 Gyr approximately, or they may have flattened out by a small amount. These conclusions are supported by some recent theoretical models by $\mathrm{Fu}$ et al. (2009), which take into account infall, star formation based on the Kennicutt law and a delayed disk formation.

\section{The Electron Temperature Gradient}

An important confirmation of the abundance gradients in photoionized nebulae comes from the expected electron temperature gradient, since the heavy elements for which radial gradients are observed are the main coolants in these objects. Therefore, a smilar, albeit inverted gradient is expected for HII regions and PN. This is in fact observed, as can be seen even in the earlier papers on this subject. Also, well defined electron temperature gradients have been measured in spiral galaxies such as NGC 300 and M101 (Bresolin et al. 2009b) and M33 (Magrini et al. 2007a). More recently, Quireza et al. (2006) have determined accurate electron temperature gradients from radio recombination lines. The average HII region gradient is $287 \pm 46 \mathrm{~K} / \mathrm{kpc}$. A somewhat higher gradient of 373 $\mathrm{K} / \mathrm{kpc}$ was obtained earlier by Deharveng et al. (2000). Similar conclusions have been obtained by Maciel et al. (2007) for planetary nebulae, for which a steeper electron temperature gradient was derived amounting to about $670 \mathrm{~K} / \mathrm{kpc}$. Fig. 3 shows both the electron temperature gradient of $\mathrm{PN}$ and the corresponding correlation with the oxygen abundances. The difference between the electron temperature gradients of HII regions (flatter) and planetary nebulae (steeper) is a strong indication that the gradients have flattened out since the PN progenitor stars have formed. 


\section{M33: A very interesting Case}

The galaxy M33 is a very interesting case, where abundance gradients have been recently measured both from HII regions and PN, apart from other objects. Rubin et al. (2008) have obtained Ne and S abundances in a sample of HII regions in M33 using Spitzer data, covering a wide range of galactocentric distances. Average gradients of $-0.058 \pm 0.014 \mathrm{dex} / \mathrm{kpc}$ and $-0.052 \pm 0.021 \mathrm{dex} / \mathrm{kpc}$ are obtained for $\mathrm{Ne} / \mathrm{H}$ and $\mathrm{S} / \mathrm{H}$, respectively. Magrini et al. (2007a, 2007b) analyzed abundances of $\mathrm{O}, \mathrm{N}$, and $\mathrm{S}$ in a sample of HII regions and derived both electron temperature and abundance gradients for this galaxy within a radius of about $7 \mathrm{kpc}$ from the galactic nucleus. The electron temperature gradient was also measured, amounting to about $570 \mathrm{~K} / \mathrm{kpc}$, corresponding to an $\mathrm{O} / \mathrm{H}$ gradient of $-0.054 \mathrm{dex} / \mathrm{kpc}$. By considering additional objects from the recent literature, it is concluded that the oxygen data cannot be fitted with a single slope, so that an inner slope of $-0.19 \mathrm{dex} / \mathrm{kpc}$ and an outer slope of $-0.04 \mathrm{dex} / \mathrm{kpc}$ are suggested. Theoretical models have been developed in which a continuous infall of gas on the disk is assumed. The models are calculated at different epochs, varying from an age of 2 Gyr to the present day, at 13.6 Gyr. According to this model, the gradients show some mild flattening with time. More recently, Magrini et al. (2009b) considered a larger PN sample and obtained a relatively weak $\mathrm{O} / \mathrm{H}$ gradient of $-0.03 \mathrm{dex} / \mathrm{kpc}$ to $-0.04 \mathrm{dex} / \mathrm{kpc}$. Similar values were measured for $\mathrm{Ne} / \mathrm{H}(-0.03$ to $-0.05 \mathrm{dex} / \mathrm{kpc})$ and $\mathrm{S} / \mathrm{H}(-0.03$ to $-0.04 \mathrm{dex} / \mathrm{kpc}$ ). The recalculated $\mathrm{O} / \mathrm{H}$ gradient for $\mathrm{HII}$ regions is similar to these values. Therefore, at face value the PN gradients are marginally steeper than the corresponding HII region gradients. However, a single slope was obtained for the whole sample, suggesting that the derived gradients are probably lower limits for the inner galaxy, since the gradients tend to flatten out at larger galactocentric distances. Moreover, PN may have progenitors with different ages, so that mixing these objects would contribute to flatten the measured slopes. This is reinforced by the fact that the inner sample, which is associated with the highest metallicities, is undesampled. A hint on this point can be obtained considering the results by Cioni (2009) on M33. She has considered a sample of well measured AGB stars, showing that the $[\mathrm{Fe} / \mathrm{H}]$ gradient is clearly steepeer in the inner parts of the galaxy, flattening out at the outer parts. Since PN and AGB stars are objects of similar ages, their gradients are expected to be similar, which reinforces the conclusion that the flatter gradients found by Magrini et al. (2009b) are lower limits.

\section{Conclusions}

From all objects considered, some tentative conclusions may be drawn: (1) Average abundance gradients are generally between $-0.03 \mathrm{dex} / \mathrm{kpc}$ and $-0.10 \mathrm{dex} / \mathrm{kpc}$, but a single value for the whole disk may be misleading. (2) Most evidences point to a flattening out of the gradients at large galactocentric distances. (3) There are some clear evidences of a flattening of the gradients near the galactic bulge. (4) The change of slope in the outer Galaxy occurs in the region around $R \simeq 10 \mathrm{kpc}$. (5) Any further change of the slope needs better data than presently available. Cepheids may be an exception. (6) There are no evidences of a steepening of the gradients at large galactocentric distances, as suggested by some theoretical models. (7) Either the gradients do not change appreciably during galactic evolution, or they flatten out at a moderate rate. (8) There are no clear evidences of a steepening of the gradients with time, as suggested by some theoretical models.

\section{Acknowledgements}

This work was partially supported by FAPESP and CNPq. 


\section{References}

Andrievsky S. M., Bersier D., Kovtyukh V. V., et al. 2002b, A\&A, 384, 140

Andrievsky S. M., Kovtyukh V. V., Luck R. E., et al. 2002a, A\&A, 381, 32

Andrievsky, S. M., Kovtyukh, V. V., Luck, R. E., et al. 2002c, A\&A, 392, 491

Andrievsky, S. M., Luck, R. E., Martin, P., \& Lépine, J. R. D. 2004, A\&A, 413, 159

Bresolin, F., Gieren, W., Kudritzki, R. P., et al. 2009b, ApJ, 700, 309

Bresolin, F., Ryan-Weber, E., et al. 2009a, ApJ, 695, 580

Cahn, J. H., Kaler, J. B., \& Stanghellini, L. 1992, A\&AS, 94, 399

Cavichia, O., Costa, R. D. D., \& Maciel, W. J. 2009, in preparation

Cescutti, G., Matteucci, F., François, P., \& Chiappini, C. 2007, A\&A, 462, 943

Chiappini, C., Matteucci, F., \& Gratton, R. 1997, ApJ, 477, 765

Chiappini, C., Matteucci, F., \& Romano, D. 2001, ApJ, 554, 1044

Chiappini, C., Romano, D., \& Matteucci, F. 2003, MNRAS, 339, 63

Cioni, M. R. 2009, A\&A, in press (astro-ph/0904.3136)

Colavitti, E., Cescutti, G., Matteucci, F., \& Murante, G. 2009, A\&A, 496, 429

Davies, B., Origlia, L., Kudritzki, R., et al. 2009, ApJ, 696, 2014

Deharveng, L., Peña, M., Caplan, J., \& Costero, R. 2000, MNRAS, 311, 329

Esteban, C., Garcia-Rojas, J., Peimbert, M., et al. 2005, ApJ, 618, L95

Freeman, K. C. 2009, IAU Symp. 254, ed. J. Andersen et al., CUP, 111

Friel, E. D., Janes, K. A., Tavarez, M., et al. 2002, AJ, 124, 2693

Fu, J., Hou, J. L., Hin, J., \& Chang, R. X. 2009, ApJ, 696, 668

Gutenkunst, S., Bernard-Salas, J., Pottasch, S. R., et al. 2008, ApJ, 680, 120

Hou, J. L., Prantzos, N., \& Boissier, S. 2000, A\&A, 362, 921

Ivezić, Z., Sesar, B., Jurić, M., et al. 2008, ApJ, 684, 2871

Lemasle, B., François, P., Piersimoni, A., et al.. 2008, A\&A, 490, 613

Luck, R. E., Gieren, W. P., Andrievsky, S. M., et al. 2003, A\&A, 401, 939

Maciel, W. J. \& Costa, R. D. D. 2009, IAU Symp. 254, ed. J. Andersen et al., CUP, electronic publication (astro-ph/0806.3443)

Maciel, W. J., Costa, R. D. D., \& Idiart, T. E. P. 2009, IAU Symp. 258, ed. E. E. Mamajek et al., electronic publication (http://abstracts.stsci.edu/IAU258/Maciel.pdf)

Maciel, W. J., Costa, R. D. D., \& Uchida, M. M. M. 2003, A\&A, 397, 667

Maciel, W. J., Lago, L. G., \& Costa, R. D. D., 2005, A\&A, 433, 127

Maciel, W. J., Lago, L. G., \& Costa, R. D. D., 2006, A\&A, 453, 587

Maciel, W. J., Quireza, C., \& Costa, R. D. D., 2007, A\&A, 463, L13

Magrini, L., Corbelli, E., \& Galli, D. 2007b, A\&A, 470, 843

Magrini, L., Sestito, P., Randich, S., \& Galli, D. 2009a, A\&A, 494, 95

Magrini, L., Stanghellini, L., \& Villaver, E. 2009b, ApJ, 696, 729

Magrini, L., Vilchez, J. M., Mampaso, A., et al., 2007a, A\&A, 470, 865

Mollá, M., Díaz, A. I. 2005, MNRAS, 358, 521

Pedicelli, S., Bono, G., Lemasle, B., et al. 2009, A\&A, in press (astro-ph/0906.3140)

Perinotto, M. \& Morbidelli, L. 2006, MNRAS, 372, 45

Pilyugin, L. S., Ferrini, F., \& Shkvarun, R. V. 2003, A\&A, 401, 557

Pottasch, S., R. \& Bernard-Salas, J. 2006, A\&A, 457, 189

Quireza, C., Rood, R. T., Bania, T. M., Balser, D. S., \& Maciel, W. J. 2006, ApJ, 653, 1226

Romaniello, M., Primas, F., \& Mottini, M. 2008, A\&A, 488, 731

Rubin, R. H., Simpson, J. P., Colgan, S. W. J., et al. 2008, MNRAS, 387, 45

Rudolph, A. L., Fich, M., Bell, G. R., et al. 2006, ApJS, 162, 346

Sestito, P., Bragaglia, A., Randich, S., et al. 2008, A\&A, 488, 943

Shaver, P. A., McGee, R. X., Newton, L. M., et al. 1983, MNRAS, 204, 53

Stanghellini, L., Guerrero, M. A., Cunha, K., et al. 2006, ApJ, 651, 898

Stanghellini, L., Shaw, R. A., \& Villaver, E. 2008, ApJ, 689, 194

Stasińska, G. 2004, In: Cosmochemistry. The melting pot of the elements. XIII Canary Islands Winter School of Astrophysics, Ed. C. Esteban, R. J. García López, et al., CUP, 115 